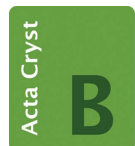

STRUCTURAL SCIENCE CRYSTAL ENGINEERING MATERIALS

ISSN 2052-5206

Received 14 October 2014

Accepted 26 February 2015

Edited by M. Dusek, Academy of Sciences of the Czech Republic, Czech Republic

Keywords: protron transfer; ferroelectric materials; incommensurate structure.

CCDC reference: 1051555

B-IncStrDB reference: 10522E4Avuq

Supporting information: this article has supporting information at journals.iucr.org/b

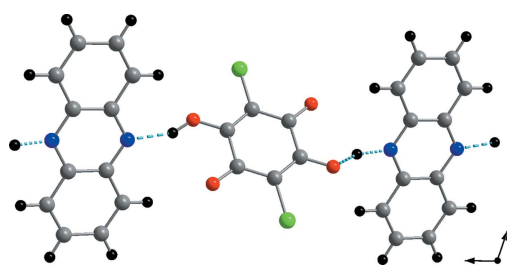

OPEN $\odot$ ACCESS

\section{Resonance-stabilized partial proton transfer in hydrogen bonds of incommensurate phenazine- chloranilic acid}

\author{
Leila Noohinejad, ${ }^{a}$ Swastik Mondal, ${ }^{\mathrm{a}, \mathrm{b}}$ Sk Imran Ali, ${ }^{\mathrm{a}}$ Somnath Dey, ${ }^{\mathrm{a}}$ Sander van \\ Smaalen $^{\mathrm{a} *}$ and Andreas Schönleber ${ }^{\mathrm{a}}$
}

aLaboratory of Crystallography, University of Bayreuth, 95440 Bayreuth, Germany, and ${ }^{\mathbf{b}}$ Max-Planck-Institut für
Kohlenforschung, Kaiser-Wilhelm-Platz 1, 45470 Mülheim an der Ruhr, Germany. *Correspondence e-mail:
smash@uni-bayreuth.de

The co-crystal of phenazine $(\mathrm{Phz})$ and chloranilic acid $\left(\mathrm{H}_{2} \mathrm{ca}\right)$ becomes ferroelectric upon cooling through the loss of inversion symmetry. Further cooling results in the development of an incommensurate ferroelectric phase, followed by a lock-in transition towards a twofold superstructure. Here we present the incommensurately modulated crystal structure of $\mathrm{Phz}-\mathrm{H}_{2} \mathrm{ca}$ at $T=$ $139 \mathrm{~K}$ with a symmetry given by the superspace group $P 2_{1}\left(\frac{1}{2} \sigma_{2} \frac{1}{2}\right) 0$ and $\sigma_{2}=$ 0.5139 . The modulation mainly affects the positions of the protons within half of the intermolecular hydrogen bonds that are responsible for the spontaneous polarization in all three low-temperature phases. Evidence for proton transfer in part of the hydrogen bonds is obtained from the correlated dependence on the phase of the modulation of the lengths of bonds involved in resonance stabilization of the acidic anion, and much smaller variations of bond lengths of atoms not involved in the resonance mechanism. Incommensurability is explained as competition between proton transfer favored for single hydrogen bonds on the basis of $\mathrm{p} K_{\mathrm{a}}$ values and avoiding unfavorable Coulomb repulsion within the lattice of the resulting ionic molecules.

\section{Introduction}

Organic compounds are of interest as ferroelectric materials, because they have a low density and they are potentially cheap to produce (Horiuchi \& Tokura, 2008). Furthermore, organic compounds offer more possibilities than inorganic compounds for designing properties. Organic materials based on hydrogen-bonded supramolecular chains with a polar space group form one class of ferroelectric materials. The co-crystal of phenazine (Phz) and 2,5-dichloro-3,6-dihydroxy-p-benzoquinone (chloranilic acid, $\mathrm{H}_{2} \mathrm{ca}$ ) is one of several recently discovered hydrogen-bonded organic ferroelectrics (Horiuchi, Ishii et al., 2005; Horiuchi et al., 2009; Kumai et al., 2006, 2012).

$\mathrm{Phz}-\mathrm{H}_{2} \mathrm{ca}$ contains chains of alternating $\mathrm{Phz}$ and $\mathrm{H}_{2} \mathrm{ca}$ molecules connected through $\mathrm{O}-\mathrm{H} \cdots \mathrm{N}$ intermolecular hydrogen bonds. At room temperature, all hydrogen bonds are equivalent by the symmetry of the centrosymmetric space group $P 2_{1} / n(Z=2)$, and the crystal of $\mathrm{Phz}-\mathrm{H}_{2} \mathrm{ca}$ is paraelectric (PE phase; Horiuchi, Ishii et al., 2005; Kumai et al., 2007). Below $T_{\mathrm{c}}^{\mathrm{I}}=253 \mathrm{~K}$ the symmetry is reduced to $P 2_{1}$ $(Z=2)$, allowing for two inequivalent hydrogen bonds. One of the two bonds exhibits partial proton transfer, which is responsible for the spontaneous polarization (FE-I phase; Horiuchi, Ishii et al., 2005; Kumai et al., 2007; Gotoh et al., 2007). Phz- $\mathrm{H}_{2} \mathrm{ca}$ has an incommensurately modulated structure between $T_{\mathrm{c}}^{\mathrm{IC}}=147 \mathrm{~K}$ and $T_{\mathrm{c}}^{\mathrm{II}}=137 \mathrm{~K}$ (FE-IC phase; Saito et al., 2006; Horiuchi et al., 2009). Below $T_{\mathrm{c}}^{\mathrm{II}}$ another ferro- 
Table 1

Experimental details.

\begin{tabular}{|c|c|}
\hline \multicolumn{2}{|l|}{ Crystal data } \\
\hline Chemical formula & $\mathrm{C}_{12} \mathrm{H}_{8} \mathrm{~N}_{2} \cdot \mathrm{C}_{6} \mathrm{H}_{2} \mathrm{Cl}_{2} \mathrm{O}_{4}$ \\
\hline$M_{\mathrm{r}}$ & 389.2 \\
\hline Crystal system, superspace group & Monoclinic, $P 2_{1}\left(\frac{1}{2} \sigma_{2} \frac{1}{2}\right) 0$ \\
\hline Temperature $(\mathrm{K})$ & 139 \\
\hline Wavevector & $\mathbf{q}=0.5 \mathbf{a}^{*}+0.5139 \mathbf{b}^{*}+0.5 \mathbf{c}^{*}$ \\
\hline$a, b, c(\AA)$ & $12.3720(2), 3.7649(5), 16.8315(2)$ \\
\hline$\beta\left({ }^{\circ}\right)$ & $107.789(7)$ \\
\hline$V\left(\AA^{3}\right)$ & $746.52(14)$ \\
\hline$Z$ & 2 \\
\hline Radiation type & Synchrotron, $\lambda=0.56 \AA$ \\
\hline$\mu\left(\mathrm{mm}^{-1}\right)$ & 0.24 \\
\hline Crystal size (mm) & $0.22 \times 0.13 \times 0.05$ \\
\hline \multicolumn{2}{|l|}{ Data collection } \\
\hline Diffractometer & Marresearch, mar165 CCD \\
\hline Absorption correction & $\begin{array}{l}\text { Empirical (using intensity measure- } \\
\text { ments) } S A D A B S \text { (Sheldrick, 2008) }\end{array}$ \\
\hline$T_{\min }, T_{\max }$ & $0.777,0.991$ \\
\hline $\begin{array}{l}\text { No. of measured, independent and } \\
\text { observed }[I>3 \sigma(I)] \text { reflections }\end{array}$ & $25001,15433,8092$ \\
\hline Main (obs, all) & 5607,5942 \\
\hline Satellites, first order (obs, all) & 2485,9491 \\
\hline$R_{\text {int }}$ & 0.019 \\
\hline$(\sin \theta / \lambda)_{\max }\left(\AA^{-1}\right)$ & 0.981 \\
\hline \multicolumn{2}{|l|}{ Refinement } \\
\hline$R\left[F^{2}>2 \sigma\left(F^{2}\right)\right], w R\left(F^{2}\right), S$ & $0.045,0.071,2.08$ \\
\hline No. of reflections & 15433 \\
\hline No. of parameters & 719 \\
\hline H-atom treatment & $\begin{array}{l}\mathrm{H} \text { atoms treated by a mixture of } \\
\text { independent and constrained } \\
\text { refinement }\end{array}$ \\
\hline$\Delta \rho_{\max }, \Delta \rho_{\min }\left(\mathrm{e} \AA^{-3}\right)$ & $0.17,-0.02$ \\
\hline Absolute structure & $\begin{array}{l}6649 \text { Friedel pairs used in the refine- } \\
\text { ment }\end{array}$ \\
\hline Absolute structure parameter & 0.5 \\
\hline
\end{tabular}

electric phase is stable that can be characterized as a twofold superstructure of the room-temperature structure (FE-II phase; Noohinejad et al., 2014).

Here we report the crystal structure of the incommensurate phase, employing the superspace formalism applied to singlecrystal X-ray diffraction data. The modulation is found to mainly affect the positions of the $\mathrm{H}$ atoms within the $\mathrm{O}-$ $\mathrm{H} \cdots \mathrm{N}$ intermolecular hydrogen bonds. Evidence for proton transfer in part of these bonds is provided by the correlated variations of bond lengths reflecting resonance stabilization of the anion. A detailed comparison of the various phases reveals that the incommensurate phase has a crystal structure intermediate between the crystal structures of the FE-I and FE-II phases. A mechanism is proposed for the sequence of phase transitions.

\section{Experimental}

\subsection{X-ray diffraction}

Single crystals of $\mathrm{Phz}-\mathrm{H}_{2} \mathrm{ca}$ were obtained by cosublimation of phenazine and chloranilic acid (Horiuchi. Ishii et al., 2005; Noohinejad et al., 2014). A diffraction experiment at $T=$ $139 \mathrm{~K}$ was performed on the same crystal as was employed in our previous study on the commensurate FE-II phase
(Noohinejad et al., 2014). X-ray diffraction data have been measured at beamline F1 of Hasylab at DESY in Hamburg, Germany, employing a MAR165 CCD detector mounted on a kappa diffractometer. The temperature of the crystal was regulated by a nitrogen gas-flow cryostat. X-ray diffraction data were collected by $\varphi$ scans and $\omega$ scans for various settings of the orientation of the crystal. To better evaluate strong and weak reflections, two measurements were performed with the same measurement strategies but with different exposure times of 20 and $160 \mathrm{~s}$, respectively. Data processing of the measured images has been carried out with the software EVAL15 (Schreurs et al., 2010) to index and extract the integrated intensities of Bragg reflections, and with $S A D A B S$ (Sheldrick, 2008) for absorption correction. The latter employed groups of equivalent reflections defined according to the point group $2 / m$, which appeared as the symmetry of the diffraction. Experimental details are given in Table 1.

Indexing of the diffraction images with EVAL15 resulted in an indexing with four integers on the basis of a monoclinic unit cell closely related to the unit cell of the FE-I phase at $160 \mathrm{~K}$ (Horiuchi, Ishii et al., 2005) together with the incommensurate modulation wavevector $\mathbf{q}^{\prime}=\left(\frac{1}{2}, \sigma_{2}^{\prime}, \frac{1}{2}\right)$, where $\sigma_{2}^{\prime}=0.4861$. However, the integration routine of EVAL15 did not accept a modulation wavevector with rational components. Therefore, the integration has been performed within the supercentered setting with $\mathbf{q}_{i}^{\prime}=\left(0, \sigma_{2}^{\prime}, 0\right)$ and centering translation $\left(\frac{1}{2}, 0, \frac{1}{2}, \frac{1}{2}\right)$ with respect to the transformed basic structure unit cell $\mathbf{A}=\mathbf{a}-\mathbf{c}, \mathbf{B}=\mathbf{b}$, and $\mathbf{C}=\mathbf{a}+\mathbf{c}$ (Stokes et al., 2011). The same setting has been employed in $S A D A B S$.

\subsection{Choice of the superspace group}

The low-temperature superstructure of the FE-II phase at $100 \mathrm{~K}$ has been described as a commensurately modulated structure with a basic structure similar to the structure at higher temperatures and the commensurate modulation wavevector $\quad \mathbf{q}_{\text {comm }}=\left(\frac{1}{2}, \frac{1}{2}, \frac{1}{2}\right)$. The superspace group $P 2_{1}\left(\frac{1}{2} \sigma_{2} \frac{1}{2}\right) 0$, with $\sigma_{2}=\frac{1}{2}$ has been found to describe the symmetry of this phase (Noohinejad et al., 2014).

Presently, the indexing with modulation wavevector $\mathbf{q}^{\prime}=\left(\frac{1}{2}, \sigma_{2}^{\prime}, \frac{1}{2}\right)$ and $\sigma_{2}^{\prime}=0.4861$ (see $\left.\$ 2.1\right)$ leads to the superspace group $P 2_{1}\left(\frac{1}{2} \sigma_{2}^{\prime} \frac{1}{2}\right) s$. The non-centrosymmetric superspace group is established by the lack of inversion symmetry of both the FE-I and FE-II phases (see $\$ 1$ ) as well as by measurements of the electrical polarization, indicating a ferroelectric state below $T_{\mathrm{c}}^{\mathrm{I}}$ (Horiuchi, Kumai \& Tokura et al., 2005). The two superspace groups appear to be alternate settings of superspace group No. 4.1.6.3 with standard setting $P 2_{1}\left(\frac{1}{2} 0 \sigma_{3}\right) 0$ (Stokes et al., 2011). The two settings can be transformed into each other by a shift of the origin. However, this would result in different coordinates of the atoms in the basic structures of the low-temperature and incommensurate phases, which is not desired. The setting with zero intrinsic translation along the fourth coordinate can also be obtained by the choice of a different modulation wavevector for the incommensurate modulation, according to 


$$
\begin{aligned}
\mathbf{q} & =\mathbf{a}^{*}+\mathbf{b}^{*}+\mathbf{c}^{*}-\mathbf{q}^{\prime} \\
& =\left(\frac{1}{2}, \sigma_{2}, \frac{1}{2}\right)
\end{aligned}
$$

with

$$
\sigma_{2}=1-\sigma_{2}^{\prime}=0.5139
$$

Diffraction data were re-indexed according to this transformation [equations (1) and (2)], and the superspace group $P 2_{1}\left(\frac{1}{2} \sigma_{2} \frac{1}{2}\right) 0$ with $\sigma_{2}=0.5139$ has been used for all refinements.

\subsection{Structure refinements}

Initial values for the parameters of the basic structure have been taken from the basic structure at $100 \mathrm{~K}$ (Noohinejad et al., 2014). Anisotropic atomic displacement parameters (ADPs) have been used for all non- $\mathrm{H}$ atoms. $\mathrm{H}$ atoms were placed at calculated positions with a bond length $d(\mathrm{C}-\mathrm{H})$ of $0.96 \AA$, and they were refined using a riding model with isotropic ADPs equal to 1.2 times the equivalent isotropic ADPs of the bonded $\mathrm{C}$ atoms. $\mathrm{H}$ atoms of the hydroxyl groups were located in the difference Fourier map. They were then shifted to positions fulfilling the restraints $d(\mathrm{O}-\mathrm{H})=$ 0.85 (2) $\AA$ and $\angle(\mathrm{C}-\mathrm{O}-\mathrm{H})=109.5$ (2) ${ }^{\circ}$ (Müller et al., 2006; Engh \& Huber, 1991), while their isotropic ADPs were restricted to 1.5 times the equivalent isotropic ADPs of the adjacent $\mathrm{O}$ atoms. Employing JANA2006 (Petricek et al., 2014), the positions of all atoms were refined with these restraints in effect. In the last step the restraints were released, resulting in a good fit to the main reflections with $R_{\mathrm{obs}}=$ 0.0412 .

Three approaches have been chosen for determination of the atomic modulation functions for the incommensurate phase. In one approach, the modulation functions of the model at $100 \mathrm{~K}$ (Noohinejad et al., 2014) were used as a starting model. The same superspace group was employed, but with $\sigma_{2}=0.5139$ instead of the commensurate value of 0.5 . The refinement converged smoothly to a good fit to the combined set of main and satellite reflections, resulting in model A (Table 1). Model A involves one harmonic wave for the displacive modulation of all atoms as well as one harmonic wave for the modulation of ADPs of all non-H atoms. The origin was fixed on the $\mathrm{Cl} 2$ atom. Inversion twins are expected to be present, because the IC phase has been reached by phase transitions, starting with the centrosymmetric PE phase at room temperature. Twinning did have a marginal effect on the refinement, while a significant deviation from equal volume fractions of the twin domains was not found. Therefore, equal volume fractions were employed for the final refinements. A model with the alternative symmetry $P 2_{1}\left(\frac{1}{2} \sigma_{2} \frac{1}{2}\right) s$ did not lead to a good fit to the data.

Starting with the same basic structure, model B was developed by assigning arbitrary but small values to the modulation parameters of the heaviest atom (chlorine). Refinements alternated with the subsequent introduction of modulation parameters for the $\mathrm{O}, \mathrm{N}, \mathrm{C}$ and $\mathrm{H}$ atoms, finally resulting in a fit to the diffraction data of equal quality as that of model A (Table 2).
Table 2

$R$ values for the different structure models.

Included are $R$ values for observed (obs; defined by $I>3 \sigma$ ) and all (all) reflections. Partial $R$ values are given for the group of main and satellite (sat) reflections. Structure model A is based on the low-temperature commensurate structure; model B has been obtained by refinement with arbitrary but small initial values for the modulation amplitudes; and model $\mathrm{C}$ is obtained after refinement of the superflip solution.

\begin{tabular}{llll}
\hline Model & A & B & C \\
\hline GOF $^{\text {obs }}$ & 2.78 & 2.78 & 2.79 \\
$R_{F}^{\text {obs }}$ (all) & 0.0449 & 0.0449 & 0.0451 \\
$R_{F}^{\text {obs }}$ (main) & 0.0411 & 0.0412 & 0.0411 \\
$R_{F}^{\text {obs }}$ (sat) & 0.1268 & 0.1266 & 0.1301 \\
$\mathrm{GOF}^{\text {all }}$ & 2.08 & 2.08 & 2.08 \\
$R_{F}^{\text {all }}$ (all) & 0.0720 & 0.0718 & 0.0720 \\
$R_{F}^{\text {all }}$ (main) & 0.0425 & 0.0425 & 0.0425 \\
$R_{F}^{\text {all }}$ (sat) & 0.3661 & 0.3634 & 0.3663 \\
No. of parameters & 719 & 719 & 719 \\
\hline
\end{tabular}

In a completely different approach, charge flipping was applied for the direct solution of the incommensurately modulated structure in superspace (Palatinus \& Chapuis, 2007; Palatinus, 2013). For the solution, the software SUPERFLIP suggested the centrosymmetric symmetry $P 2_{1} / n\left(\frac{1}{2} \sigma_{2} \frac{1}{2}\right) 00$. Since we knew that the modulation is noncentrosymmetric, we have chosen the superspace group $P 2_{1}\left(\frac{1}{2} \sigma_{2} \frac{1}{2}\right) 0$. JANA2006 was subsequently used to extract the basic structure positions and values of the first-order harmonics of the displacive modulation functions for all non- $\mathrm{H}$ atoms. Atoms were then named to match Fig. 1. For this model the basic structure coordinates were refined against all reflections, resulting in $R_{F}=0.3046, R_{F}^{\text {main }}=0.2901$ and $R_{F}^{\text {sat }}=0.6184$. $\mathrm{H}$ atoms were added at calculated positions near $\mathrm{C}$ atoms as in model $\mathrm{A}$. Refinement of the atomic coordinates within the riding model resulted in $R_{F}=0.3037$, $R_{F}^{\text {main }}=0.2892$ and $R_{F}^{\text {sat }}=0.6174$. Subsequent refinement of anisotropic ADPs of the non-H atoms resulted in $R_{F}=0.0680, R_{F}^{\text {main }}=0.0430$ and $R_{F}^{\text {sat }}=0.6081$. H atoms of the hydroxyl groups were located in the difference-Fourier map and treated as in model A. Refinement of the restrained model gave $R_{F}=0.0672, R_{F}^{\text {main }}=0.0427$ and $R_{F}^{\text {sat }}=0.5993$. Refinement of the free model gave $R_{F}=0.0666$,

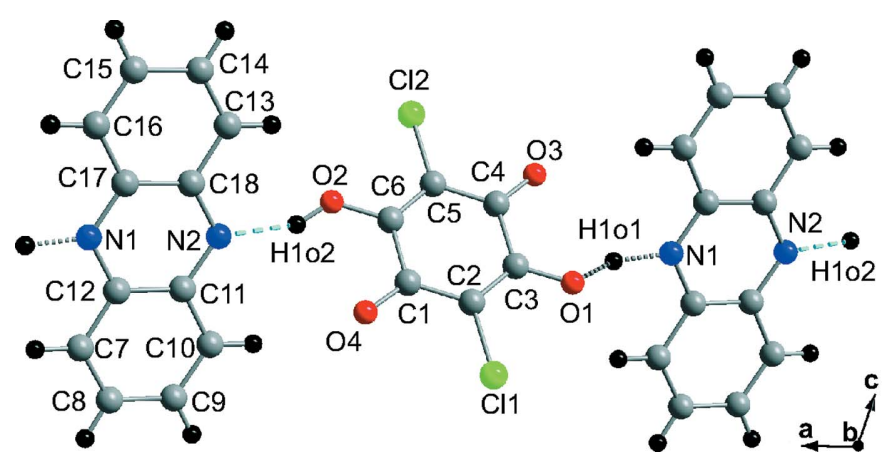

Figure 1

Phenazine $\mathrm{C}_{12} \mathrm{H}_{8} \mathrm{~N}_{2}$ and chloranilic acid $\mathrm{C}_{6} \mathrm{Cl}_{2} \mathrm{H}_{2} \mathrm{O}_{4}$ with the atom labels as employed in the present work. 
$R_{F}^{\text {main }}=0.0420$ and $R_{F}^{\text {sat }}=0.5998$. Small values were applied to the displacive modulation functions of the $\mathrm{H}$ atoms. Refinement of the modulated structure resulted in $R_{F}=0.0472, \quad R_{F}^{\text {main }}=0.0412$ and $R_{F}^{\text {sat }}=0.1787$. Finally, modulation parameters were introduced for the ADPs of the non- $\mathrm{H}$ atoms, resulting in the final fit of model $\mathrm{C}$ to the diffraction data as given in Table 2.

\section{Discussion}

\subsection{The structure model}

The final fit to the diffraction data is excellent for the main reflections (Table 1). The rather high value of $R_{F}^{\text {obs }}($ sat $)=0.127$ can be completely explained by the weakness of the satellite reflections and the resulting values for $R_{\sigma}$ (sat) $=0.151$, representing the average standard uncertainty over intensity, and $R_{\text {int }}(\mathrm{sat})=0.129$ for averaging satellite reflections.

Model A and model B give the same fit to the diffraction data (Table 2). Although modulation parameters are different, these two models are completely equivalent. They differ from each other by a phase shift (Fig. 2). Further support for model A comes from difference-Fourier maps obtained after refinements of model A and of a similar model without the acidic $\mathrm{H}$ atoms (see the supporting information). Model $\mathrm{C}$ has been obtained by solving the modulated structure by charge flipping in superspace. The modulation of model $\mathrm{C}$ is different from the modulations in models $\mathrm{A}$ and $\mathrm{B}$, but $R_{F}^{\mathrm{obs}}$ (sat) is clearly higher for model $\mathrm{C}$ than for the other two models (Table 2). Therefore, model $\mathrm{C}$ provides a less good description of the modulation than models A and B do. Difficulties in obtaining the correct structure model by charge flipping are probably related to the pseudo-symmetry of the structure, with deviations from inversion symmetry being mainly the result of rearrangements of $\mathrm{H}$ atoms.

These properties provide strong support that model A (as well as the equivalent model $\mathrm{B}$ ) is the correct model for the

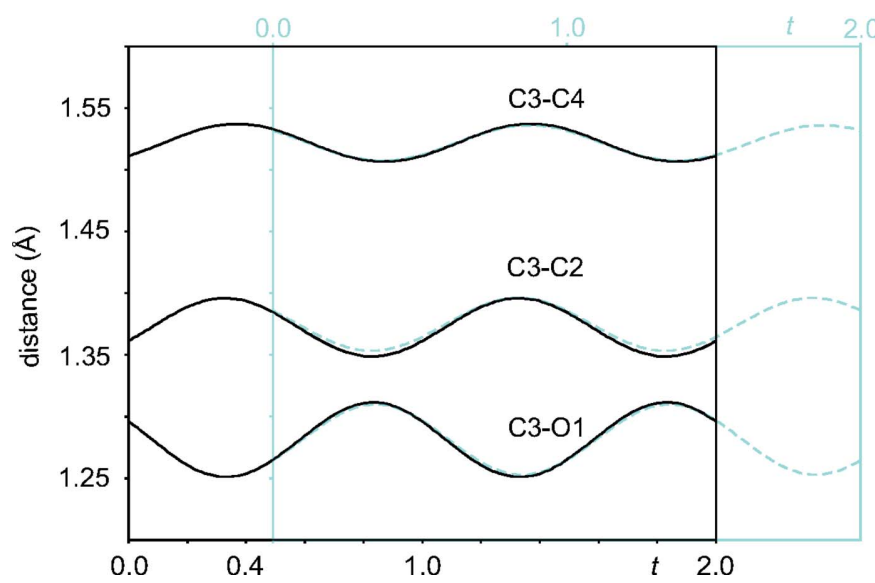

Figure 2

Interatomic distances $(\AA)$ as a function of phase $t$ of the modulation. The $t$ plot for model B (in blue) is superimposed onto the $t$ plot for model A (in black), after application of a phase shift of -0.5139 in $t$ to model B.
Table 3

Geometry of the intermolecular hydrogen bonds $\mathrm{O} 1-\mathrm{H} 1 \mathrm{o} 1 \cdots \mathrm{N} 1$ and $\mathrm{O} 2-\mathrm{H} 1 \mathrm{o} 2 \cdots \mathrm{N} 2$ at different temperatures corresponding to the FE-I, FE-IC and FE-II phases, respectively.

Interatomic distances are given in $\AA$ and bond angles in degrees. (Max-min) provides the difference between the maximum $(\max )$ and minimum (min) separation depending on the phase $t$ of the modulation in the FE-IC phase. The mean gives the value averaged over $t$. Standard uncertainties are given in parentheses.

\begin{tabular}{|c|c|c|c|c|}
\hline & $170 \mathrm{~K} \dagger$ & $139 \mathrm{~K}$ & & $100 \mathrm{~K} \ddagger$ \\
\hline & Distance & Distance & Max-min & Distance \\
\hline $\mathrm{O} 1-\mathrm{H} 1 \mathrm{o} 1$ & $1.02(4)$ & 1.44 (2) (mean) & 0.25 & 0.943 (15) (A) \\
\hline & & $1.32(2)(\min )$ & & 1.609 (15) (B) \\
\hline & & $1.57(2)(\max )$ & & $1.066(14)(\mathrm{C})$ \\
\hline & & & & 1.467 (14) (D) \\
\hline $\mathrm{O} 2-\mathrm{H} 1 \mathrm{o} 2$ & $0.73(2)$ & 0.91 (2) (mean) & 0.06 & 0.863 (15) (A) \\
\hline & & $0.88(2)(\min )$ & & 0.796 (15) (B) \\
\hline & & $0.94(2)(\max )$ & & 0.840 (14) (C) \\
\hline & & & & 0.815 (13) (D) \\
\hline $\mathrm{H} 1 \mathrm{o} 1-\mathrm{N} 1^{\mathrm{i}}$ & $1.66(4)$ & 1.320 (14) (mean) & 0.42 & $1.879(15)(\mathrm{A})$ \\
\hline & & $1.11(2)(\min )$ & & 1.027 (15) (B) \\
\hline & & $1.53(2)(\max )$ & & $1.700(14)(\mathrm{C})$ \\
\hline & & & & 1.205 (14) (D) \\
\hline $\mathrm{H} 1 \mathrm{o} 2-\mathrm{N} 2^{\mathrm{ii}}$ & $2.15(2)$ & 1.945 (2) (mean) & 0.05 & 1.908 (14) (A) \\
\hline & & $1.92(2)(\min )$ & & $2.121(14)(\mathrm{B})$ \\
\hline & & $1.97(2)(\max )$ & & 1.944 (14) (C) \\
\hline & & & & 2.084 (14) (D) \\
\hline $\mathrm{O} 1-\mathrm{N} 1^{\mathrm{i}}$ & $2.6446(16)$ & 2.629 (2) (mean) & 0.07 & $2.6976(14)(\mathrm{A})$ \\
\hline & & $2.586(2)(\min )$ & & $2.5736(14)(\mathrm{B})$ \\
\hline & & $2.672(2)(\max )$ & & $2.5974(42)(\mathrm{D})$ \\
\hline & & & & $2.6726(14)(\mathrm{C})$ \\
\hline $\mathrm{O} 2-\mathrm{N} 2^{\mathrm{ii}}$ & $2.7722(16)$ & 2.763 (2) (mean) & 0.09 & $2.7086(15)(\mathrm{A})$ \\
\hline & & $2.715(3)(\min )$ & & $2.8251(15)(\mathrm{B})$ \\
\hline & & $2.811(3)(\max )$ & & $2.7264(15)(\mathrm{C})$ \\
\hline & & & & $2.8062(15)(\mathrm{D})$ \\
\hline $\mathrm{O} 1-\mathrm{H} 1 \mathrm{o} 1-\mathrm{N} 1^{1}$ & $159(3)$ & 144.8 (15) (mean) & 9.9 & $143.8(11)(\mathrm{A})$ \\
\hline & & 139.5 (14) (min) & & 154.4 (11) (B) \\
\hline & & $149.7(16)(\max )$ & & 149.3 (10) (C) \\
\hline & & & & 152.6 (10) (D) \\
\hline $\mathrm{O} 2-\mathrm{H} 1 \mathrm{o} 2-\mathrm{N} 2^{\mathrm{ii}}$ & $145(2)$ & 149.2 (18) (mean) & 5.4 & $153.7(11)(\mathrm{A})$ \\
\hline & & 146.4 (17) (min) & & 147.5 (12) (B) \\
\hline & & $152.0(18)(\max )$ & & 154.5 (11) (C) \\
\hline & & & & $147.60(12)(\mathrm{D})$ \\
\hline
\end{tabular}

Symmetry codes for $\mathrm{N} 1$ and $\mathrm{N} 2$ in the structure model at $T=139 \mathrm{~K}$ are: (i) $x-1, y, z$; (ii) $x, y+1, z . \quad \dagger$ From Gotoh et al. (2007). ‡ From Noohinejad et al. (2014); the labels $\mathrm{A}, \mathrm{B}, \mathrm{C}$ and $\mathrm{D}$ refer to the four molecular chains, which have become independent in the crystal structure at low temperatures.

modulated crystal structure of the incommensurate phase. In view of these results, we have restricted the analysis to model A.

\subsection{Resonance-stabilized proton transfer}

The modulated structure of the incommensurate phase of $\mathrm{Phz}-\mathrm{H}_{2} \mathrm{ca}$ has been successfully determined at a temperature of $139 \mathrm{~K}$. The magnitudes of the modulation amplitudes of the atoms reveal that the major effect of the modulation is a displacive modulation of the $\mathrm{H}$ atom of one of the two hydrogen bonds in which each molecule is involved in. This feature is in complete agreement with the crystal structures of the FE-I and FE-II phases, where also one half of the hydrogen bond is involved in the distortions of the structure.

More precisely, the crystal structure of the FE-I phase contains one crystallographically independent molecule $\mathrm{H}_{2} \mathrm{ca}$ with two independent $\mathrm{O}$ atoms involved in $\mathrm{O}-\mathrm{H} \cdots \mathrm{N}$ 
hydrogen bonds, denoted by $\mathrm{O} 1$ and $\mathrm{O} 2$ (Gotoh et al., 2007). The basic structure of the FE-IC phase is the same as the crystal structure of the FE-I phase, so that the FE-IC phase contains modulated atoms $\mathrm{O} 1$ and $\mathrm{O} 2$. Finally, the FE-II phase represents a twofold superstructure of the structure of the FEI phase. Together with a reduction of the point symmetry to triclinic, this results in four crystallographically independent molecules $\mathrm{H}_{2}$ ca with atoms $\mathrm{O} 1 A$ through to $\mathrm{O} 1 D$ derived from $\mathrm{O} 1$, and atoms $\mathrm{O} 2 A$ through to $\mathrm{O} 2 D$ derived from $\mathrm{O} 2$ (Noohinejad et al., 2014). In all three phases, the hydrogen bonds $\mathrm{O} 2-\mathrm{H} 1 \mathrm{o} 2 \cdots \mathrm{N} 2$ are not involved in superstructure formation. For the FE-IC structure, Table 3 and Fig. 3(a) show that bond lengths involving the $\mathrm{O} 2, \mathrm{H} 1 \mathrm{o} 2$ and $\mathrm{N} 2$ atoms exhibit only a weak dependence on phase $t$ of the modulation. For the FE-I and FE-II structures this property has been previously determined by Gotoh et al. (2007) and Noohinejad et al. (2014), and it is summarized in Table 3. Therefore, the hydrogen bonds $\mathrm{O} 2-\mathrm{H} 1 \mathrm{o} 2 \cdots \mathrm{N} 2$ do not play a direct role in the ferroelectricity of this compound.

The largest variation in bond lengths within the FE-IC phase is found for the hydrogen bond $\mathrm{O} 1-\mathrm{H} 1 \mathrm{o} 1 \cdots \mathrm{N} 1$, with a variation of $\Delta d(\mathrm{O} 1-\mathrm{H} 1 \mathrm{o} 1)=0.25 \AA$ and $\Delta d(\mathrm{~N} 1-\mathrm{H} 1 \mathrm{o} 1)=$ $0.42 \AA$ (Table 3 ). All other bonds are much less affected by the modulation, with a maximum variation of $0.06 \AA$ for $\mathrm{C} 3-\mathrm{O} 1$ in $\mathrm{H}_{2}$ ca and of $0.019 \AA$ for $\mathrm{C} 14-\mathrm{C} 15$ in $\mathrm{Phz}$ (see the supporting information). The next largest variations of bond lengths are found for $\mathrm{C} 3-\mathrm{C} 2, \mathrm{C} 1-\mathrm{C} 2$ and $\mathrm{C} 1-\mathrm{O} 4$ (Table 4). These bonds are precisely those involved in resonance stabilization of the $\mathrm{Hca}^{-}$ion, as it is obtained after transfer of the proton within the $\mathrm{O} 1-\mathrm{H} 1 \mathrm{o} 1 \cdots \mathrm{N} 1$ hydrogen bond. Further evidence for this interpretation comes from $t$-plots (Fig. 3), which show that an elongation of the $\mathrm{O} 1-\mathrm{H} 1 \mathrm{o} 1$ bond (interpreted as proton transfer) correlates with an elongation of the $\mathrm{C} 3-\mathrm{C} 2$ and $\mathrm{C} 1-\mathrm{O} 4$ bonds, for which resonance represents the admixture of single-bond character into these formally double bonds (Fig. 4). Concomitantly, $\mathrm{C} 1-\mathrm{C} 2$ and C3-O1 have become shorter due to the admixture of doublebond character into formally single bonds. A similar variation of bond lengths is found in the crystal structure of the FE-II
Table 4

Selected bond lengths $(\AA)$ at different temperatures corresponding to the FE-I, FE-IC and FE-II phases, respectively.

(Max-min) provides the difference between maximum $(\max )$ and minimum (min) separation depending on phase $t$ of the modulation in the FE-IC phase. The mean gives the value averaged over $t$. Standard uncertainties are given in parentheses.

\begin{tabular}{|c|c|c|c|c|}
\hline & \multirow{2}{*}{$\begin{array}{l}170 \mathrm{~K} \dagger \\
\text { Distance }\end{array}$} & \multicolumn{2}{|l|}{$139 \mathrm{~K}$} & \multirow{2}{*}{$\begin{array}{l}100 \mathrm{~K} \ddagger \\
\text { Distance }\end{array}$} \\
\hline & & Distance & Max-min & \\
\hline \multirow[t]{4}{*}{$\mathrm{C} 3-\mathrm{O} 1$} & $1.2923(13)$ & 1.281 (2) (mean) & 0.060 & $1.3200(14)(\mathrm{A})$ \\
\hline & & $1.251(2)(\mathrm{min})$ & & $1.2536(14)(B)$ \\
\hline & & $1.311(2)(\max )$ & & $1.3054(14)(\mathrm{C})$ \\
\hline & & & & $1.2676(14)(\mathrm{D})$ \\
\hline \multirow[t]{4}{*}{$\mathrm{C} 6-\mathrm{O} 2$} & $1.3204(13)$ & 1.312 (3) (mean) & 0.004 & $1.3133(15)(\mathrm{A})$ \\
\hline & & $1.310(2)(\mathrm{min})$ & & $1.3204(15)(\mathrm{B})$ \\
\hline & & $1.314(2)(\max )$ & & $1.3118(14)(\mathrm{C})$ \\
\hline & & & & $1.3214(14)(\mathrm{D})$ \\
\hline \multirow[t]{4}{*}{$\mathrm{C} 4-\mathrm{O} 3$} & $1.2269(15)$ & 1.219 (2) (mean) & 0.008 & $1.2243(14)(\mathrm{A})$ \\
\hline & & $1.215(2)(\min )$ & & $1.2211(14)(\mathrm{B})$ \\
\hline & & $1.223(2)(\max )$ & & $1.2248(14)(\mathrm{C})$ \\
\hline & & & & $1.2201(14)(\mathrm{D})$ \\
\hline \multirow[t]{4}{*}{$\mathrm{C} 1-\mathrm{O} 4$} & $1.2291(15)$ & 1.221 (2) (mean) & 0.026 & $1.2183(14)(\mathrm{A})$ \\
\hline & & $1.208(2)(\min )$ & & $1.2385(14)(\mathrm{B})$ \\
\hline & & $1.234(2)(\max )$ & & $1.2210(14)(\mathrm{C})$ \\
\hline & & & & $1.2355(14)(\mathrm{D})$ \\
\hline \multirow[t]{4}{*}{$\mathrm{C} 1-\mathrm{C} 2$} & $1.4404(15)$ & 1.428 (3) (mean) & 0.043 & $1.4590(8)(\mathrm{A})$ \\
\hline & & $1.406(3)(\mathrm{min})$ & & 1.4114 (7) (B) \\
\hline & & 1.449 (3) (max) & & 1.4496 (8) (C) \\
\hline & & & & 1.4207 (8) (D) \\
\hline \multirow[t]{4}{*}{$\mathrm{C} 2-\mathrm{C} 3$} & $1.3713(16)$ & 1.372 (3) (mean) & 0.047 & 1.3517 (9) (A) \\
\hline & & $1.349(3)(\min )$ & & 1.3949 (9) (B) \\
\hline & & $1.396(3)(\max )$ & & $1.3622(9)(\mathrm{C})$ \\
\hline & & & & $1.3843(9)(\mathrm{D})$ \\
\hline \multirow[t]{4}{*}{$\mathrm{N} 1-\mathrm{C} 12$} & $1.3493(13)$ & 1.342 (2) (mean) & 0.006 & $1.3451(11)(\mathrm{A})$ \\
\hline & & 1.339 (2) (min) & & 1.3465 (11) (B) \\
\hline & & $1.345(2)(\max )$ & & $1.3468(14)(\mathrm{C})$ \\
\hline & & & & $1.3443(12)(\mathrm{D})$ \\
\hline \multirow[t]{4}{*}{$\mathrm{N} 1-\mathrm{C} 17$} & $1.3472(17)$ & 1.344 (2) (mean) & 0.005 & $1.3490(14)(\mathrm{A})$ \\
\hline & & $1.342(2)(\mathrm{min})$ & & 1.3505 (14) (B) \\
\hline & & $1.347(2)(\max )$ & & $1.3464(14)(\mathrm{C})$ \\
\hline & & & & $1.3526(14)(\mathrm{D})$ \\
\hline
\end{tabular}

$\dagger$ From Gotoh et al. (2007). $\ddagger$ From Noohinejad et al. (2014).

phase (Table 4). The results support the model of partial proton transfer (see \$3.3).

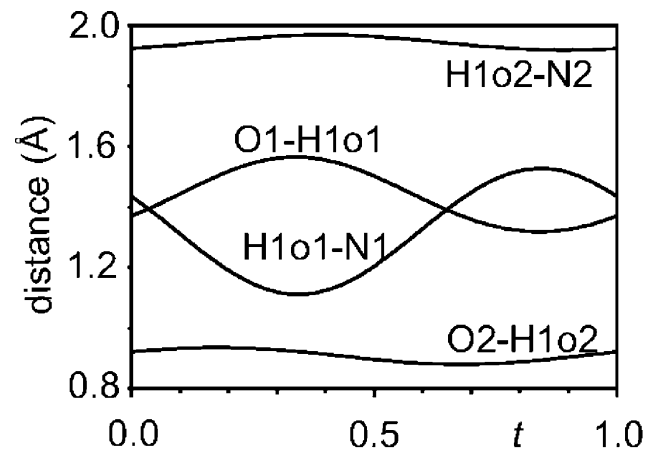

Figure 3

(a)

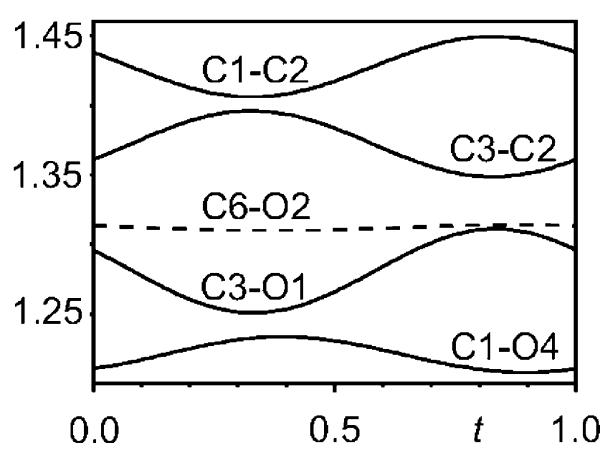

(b)

Selected interatomic distances $(\AA)$ as a function of phase $t$ of the modulation. (a) The $\mathrm{O}-\mathrm{H}$ and $\mathrm{N}-\mathrm{H}$ distances within the two hydrogen bonds. (b) $\mathrm{C}-\mathrm{C}$ and $\mathrm{C}-\mathrm{O}$ distances of the resonance system stabilizing the $\mathrm{Hca}^{-}$anion, as well as the $\mathrm{C} 6-\mathrm{O} 2$ distance not involved in resonance. Notice the different length scale on the vertical axes for panels $(a)$ and $(b)$.

3.3. The ferroelectric phase transitions

Ferroelectricity in $\mathrm{Phz}-\mathrm{H}_{2} \mathrm{ca}$ at low temperatures is the result of intermolecular proton transfer within the $\mathrm{O} 1-\mathrm{H} 1 \mathrm{o} 1 \cdots \mathrm{N} 1$ hydrogen bonds (Horiuchi, Kumai \& Tokura, 2005; Kumai et al., 2007, 2012; Gotoh et al., 2007; Noohinejad et al., 2014). Consideration of the positions of the $\mathrm{H}$ atoms within the $\mathrm{O} 1-\mathrm{H} 1 \mathrm{o} 1 \cdots \mathrm{N} 1$ hydrogen bonds of the crystal structures of the three phases leads to the following model for the phase transitions. 
At room temperature (PE phase) all hydrogen bonds are equivalent by symmetry of the centrosymmetric space group. Consequently, any dipole moment of the $\mathrm{O} 1-\mathrm{H} 1 \mathrm{o} 1 \cdots \mathrm{N} 1$ hydrogen bond will be perfectly compensated by a dipole moment of the $\mathrm{O} 2-\mathrm{H} 1 \mathrm{o} 2 \cdots \mathrm{N} 2$ hydrogen bond on the same molecule that points in the opposite direction, because $\mathrm{O} 1$ and $\mathrm{O} 2$ are related by the inversion center. The ferroelectric phase transition towards the FE-I phase is characterized by loss of inversion symmetry. The $\mathrm{O} 2-\mathrm{H} 1 \mathrm{o} 2$ remains short and should be interpreted as a covalent $\mathrm{O}-\mathrm{H}$ bond that acts as a hydrogen-bond donor towards N2 (Table 3). The O1-H1o1 bond is clearly elongated compared with a covalent bond, but it is not completely broken. The $\mathrm{N} 1-\mathrm{H} 1 \mathrm{o} 1$ distance is clearly shorter than in the PE phase, but it is not yet the distance of $\sim 1.03 \AA$ of a covalent $\mathrm{N}-\mathrm{H}$ bond. Therefore, it can be concluded that this structure exhibits partial proton transfer within the $\mathrm{O} 1-\mathrm{H} 1 \mathrm{o} 1 \cdots \mathrm{N} 1$ hydrogen bonds.

The low-temperature FE-II has four crystallographically independent $\mathrm{O} 1-\mathrm{H} 1 \mathrm{o} 1 \cdots \mathrm{N} 1$ hydrogen bonds. The partial proton transfer of the FE-I phase is replaced in the FE-II phase by complete proton transfer in one half of these hydrogen bonds ( $\mathrm{B}$ and $\mathrm{D}$ ), and the absence of proton transfer in the other half (A and C) (Table 3). The FE-I phase transfers into the FE-II phase via the intermediate FE-IC phase. Structurally, the FE-IC phase appears intermediate between the high- and low-temperature ferroelectric phases too. The incommensurate modulations represents a modulation of the $\mathrm{O} 1-\mathrm{H} 1 \mathrm{o} 1 \cdots \mathrm{N} 1$ hydrogen bond between one with almost full proton transfer and one which can be characterized as almost no proton transfer (Table 3 and Fig. 3a). Despite an incommensurability of the FE-IC phase, it appears that - on average - one quarter of the hydrogen bonds has full proton transfer in both the FE-IC and FE-II phases, while half of the hydrogen bonds in the FE-I phase are affected by partial proton transfer. These similarities might explain the only marginal effects of the ferroelectric incommensurate and lock-in transitions on the macroscopic electric dipole moment (Horiuchi et al., 2009).

\section{Conclusions}

The incommensurately modulated structure of the ferroelectric incommensurate (FE-IC) phase of $\mathrm{Phz}-\mathrm{H}_{2}$ ca has been successfully determined. It is shown that this structure is

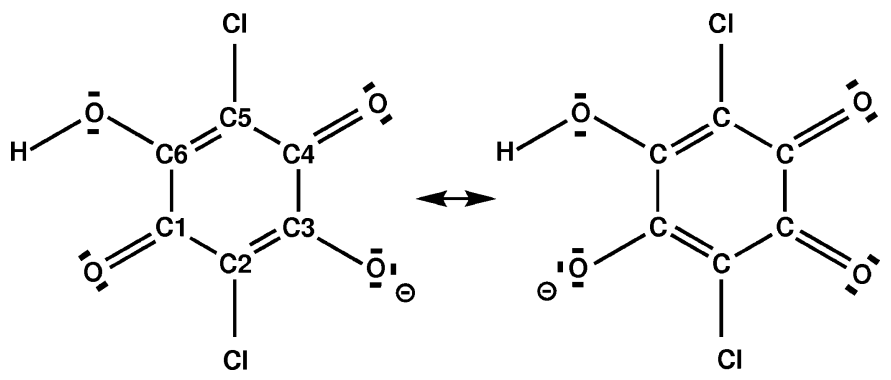

Figure 4

Schematic representation of resonance within the anion $\mathrm{Hca}^{-}$of chloranilic acid. intermediate between the ferroelectric FE-I and FE-II phases. Half of the intermolecular hydrogen bonds exhibit partial proton transfer within the FE-I phase. This becomes an incommensurate variation between strong and very weak proton transfer within the FE-IC phase, while in the FE-II phase the active half of the hydrogen bonds splits into a hydrogen bond with complete proton transfer and one without proton transfer. Strong support for proton transfer in part of the hydrogen bonds has been obtained through the variations of the lengths of precisely those bonds that are involved in resonance stabilization of the $\mathrm{Hca}^{-}$ion (\$3.2). Proton transfer in only part of the hydrogen bonds has been explained as the result of Coulomb interactions between the resulting ionic species (Kumai et al., 2012). Proton transfer is in line with the acidities of the two molecules with $\mathrm{p} K_{\mathrm{a} 1}=1.23$ for the proton acceptor $\mathrm{Phz}$, and $\mathrm{p} K_{\mathrm{a} 1}=0.76$ for the proton donor $\mathrm{H}_{2} \mathrm{ca}$ (Albert \& Phillips, 1956; Molcanov \& Prodic, 2010). One could thus suggest that the incommensurability will be the result of competition between the inclination towards proton transfer of single hydrogen bonds and avoiding unfavorable Coulomb repulsion within the crystalline lattice of molecules.

\section{Acknowledgements}

Single crystals were grown by Alfred Suttner at the Laboratory of Crystallography in Bayreuth. The help of Carsten Paulmann with diffraction experiments with synchrotron radiation at beamline F1 of Hasylab at DESY, Hamburg, is gratefully acknowledged. Beamtime has been awarded under proposal No. II-20100019. The research of LN has been made possible through financial support by the German Academic Exchange Service (DAAD).

\section{References}

Albert, A. \& Phillips, J. N. (1956). J. Chem. Soc. pp. 1294-1304.

Engh, R. A. \& Huber, R. (1991). Acta Cryst. A47, 392-400.

Gotoh, K., Asaji, T. \& Ishida, H. (2007). Acta Cryst. C63, o17-o20.

Horiuchi, S., Ishii, F., Kumai, R., Okimoto, Y., Tachibana, H., Nagaosa, N. \& Tokura, Y. (2005a). Nature Mater. 4, 163-166.

Horiuchi, S., Kumai, R. \& Tokura, Y. (2005). J. Am. Chem. Soc. 127, 5010-5011.

Horiuchi, S., Kumai, R. \& Tokura, Y. (2009). J. Mater. Chem. 19, 4421-4434.

Horiuchi, S. \& Tokura, Y. (2008). Nature Mater. 7, 357-366.

Kumai, R., Horiuchi, S., Fujioka, J. \& Tokura, Y. (2012). J. Am. Chem. Soc. 134, 1036-1046.

Kumai, R., Horiuchi, S., Okimoto, Y. \& Tokura, Y. (2006). J. Chem. Phys. 125, 084715.

Kumai, R., Horiuchi, S., Sagayama, H., Arima, T. H., Watanabe, M., Noda, Y. \& Tokura, Y. (2007). J. Am. Chem. Soc. 129, 12920-12921. Molcanov, K. \& Kojic Prodic, B. (2010). CrystEngComm, 12, 925-939.

Müller, P., Herbst-Irmer, R., Spek, A. L., Schneider, T. R. \& Sawaya, M. R. (2006). Crystal Structure Refinement. A Crystallographers Guide to SHELXL. Oxford University Press.

Noohinejad, L., Mondal, S., Wölfel, A., Ali, S. I., Schönleber, A. \& van Smaalen, S. (2014). J. Chem. Crystallogr. 44, 387-393.

Palatinus, L. (2013). Acta Cryst. B69, 1-16.

Palatinus, L. \& Chapuis, G. (2007). J. Appl. Cryst. 40, 786-790.

Petricek, V., Dusek, M. \& Palatinus, L. (2014). Z. Kristallogr. 229, 345-352. 


\section{research papers}

Saito, K., Amano, M., Yamamura, Y., Tojo, T. \& Atake, T. (2006). J. Phys. Soc. Jpn, 75, 033601.

Schreurs, A. M. M., Xian, X. \& Kroon-Batenburg, L. M. J. (2010). J. Appl. Cryst. 43, 70-82.
Sheldrick, G. M. (2008). SADABS, Version 2008/1. University of Göttingen.

Stokes, H. T., Campbell, B. J. \& van Smaalen, S. (2011). Acta Cryst. A67, 45-55. 